\title{
Transmission Electron Microscopy on Memristive Devices: An Overview
}

\author{
Julian Strobel, Krishna Kanth Neelisetty ${ }^{1}$, Venkata Sai Kiran Chakravadhanula ${ }^{1}$, Lorenz Kienle * \\ Kiel University, Faculty of Engineering, Institute for Materials Science, Kiel 24143, Germany \\ ${ }^{1}$ Karlsruhe Institute of Technology, Institute of Nanotechnology, Hermann-von-Helmholtz-Platz 1, \\ Eggenstein-Leopoldshafen 76344, Germany
}

\author{
*Correspondence to: \\ Kienle L, \\ Tel: +49-431-880-6196 \\ Fax: +49-431-880-6178 \\ E-mail: Ik@tf.uni-kiel.de \\ Received December 23, 2016 \\ Revised December 25, 2016 \\ Accepted December 25, 2016
}

\begin{abstract}
This communication is to elucidate the state-of-the-art of techniques necessary to gather information on a new class of nanoelectronic devices known as memristors and related resistive switching devices, respectively. Unlike classical microelectronic devices such as transistors, the chemical and structural variations occurring upon switching of memristive devices require cutting-edge electron microscopy techniques. Depending on the switching mechanism, some memristors call for the acquisition of atomically resolved structural data, while others rely on atomistic chemical phenomena requiring the application of advanced X-ray and electron spectroscopy to correlate the real structure with properties. Additionally, understanding resistive switching phenomena also necessitates the application not only of pre- and post-operation analysis, but also during the process of switching. This highly challenging in situ characterization also requires the aforementioned techniques while simultaneously applying an electrical bias. Through this review we aim to give an overview of the possibilities and challenges as well as an outlook onto future developments in the field of nanoscopic characterization of memristive devices.
\end{abstract}

Key Words: Memristor, Resistive switching, Transmission electron microscopy, Spectroscopy

\section{INTRODUCTION}

As Moore's law is reaching physical limitations, novel approaches in computing are being pursued. Among them is the realization of artificial neural networks that aim to mimic the human brain's capability in fields like pattern recognition and massive parallel computing all the while maintaining ultralow power consumption. These networks can be put into effect by making use of memristive devices (Hasegawa et al., 2012; Mazumder et al., 2012; Xia, 2011). These can be understood as the technological equivalent of synapses in the human brain and are essentially electronic components which can change their electrical resistance repeatedly and reversibly over billions of cycles, ideally in a non-binary fashion over a large On/Off ratio. The scope of this communication is to illustrate in a comprehensive fashion how these devices can be characterized in transmission electron microscopes
(TEMs) and what particular challenges occur which may call for extension of the present state-of-the-art in electron microscopy and sample preparation. Ideally, research on memristors brings together scientists from both the fields of microscopy as well as device conceptualization and fabrication.

The technological relevance of memristors is based on the increasing need for their aforementioned advantages. Arithmetic-logical operations are the stronghold of classical transistor-based machines. They, however, fall short in increasingly important tasks involving machine learning or machine intelligence where classification and regression require the machine to categorize certain inputs according to specific rules. In particular, this includes the distinction of simple objects like traffic signs but also much more complex tasks such as facial recognition. On top of that, optimization and prediction of data pose computational challenges for

(a) This is an open-access article distributed under the terms of the Creative Commons Attribution Non-Commercial License (http://creativecommons.org/licenses/by-nc/4.0) which permits unrestricted noncommercial use, distribution, and reproduction in any medium, provided the original work is properly cited.

Copyrights @ 2016 by Korean Society of Microscopy 
which memristor-based logic is likely most suitable to solve. The seemingly obvious analogy with transistors in terms of computation fails with respect to the very different complexity of TEM analyses on both systems. Complex to fabricate on the one hand, transistors are comparably easy objects for nanoanalysis. In general, structures in transistors are large enough to be straightforwardly distinguishable in TEM. The current technology node as denoted by the International Technology Roadmap for Semiconductors (ITRS) utilizes structures as small as $14 \mathrm{~nm}$ which is easily resolvable in virtually all TEMs.

Aside from challenges by the small dimensions of memristive components of functional devices, further complication arises from their partially unknown working principles. Where e.g., transistors are well understood and nanoscale analysis merely confirms fabrication procedures and microscopic structure, memristive devices require TEM analysis to uncover the working principle behind them, thus their analysis poses a much greater challenge. The general aim, of course, is to measure whatever influences the present resistive state of the memristor. If the present state and its parameters are well understood, analysis of any kind becomes much more straightforward. On the other hand, as fabrication technology is already well developed for microelectronics, research in the field of memristors benefits greatly from the experience of the past 35 years of transistor research.

Experimental equipment for microscopy has advanced rapidly over the past decades as well. Development of microscopes, detectors and sample preparation equipment made a gigantic leap towards today's aberration-corrected monochromated electron microscopy, with the capability for excellent chemical analysis by multiple sector energy dispersive X-ray spectroscopy (EDX) and highly precise electron energyloss spectroscopy (EELS) detectors. Although high-end microscopes may not be omnipresent, the availability of access to shared user facilities has eased the possibility of highend characterization.

One aspect that is often lacking, however, is the supply with reference and database values for comparison, especially when it comes to EEL spectra. Also, the link between structural and chemical information gathered by TEM analysis and macroscopic physical properties such as the relative permittivity or electrical and ionic conductivities is often unclear.

Generally, memristive devices can be distinguished by their working principle, cf. Fig. 1. The distinction can be made between electrochemical metallization (ECM), cells valence

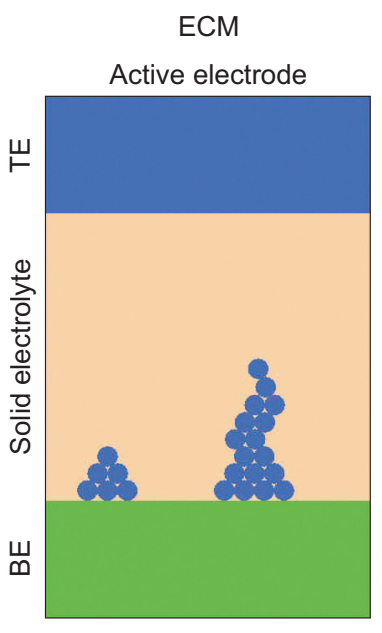

Inactive electrode

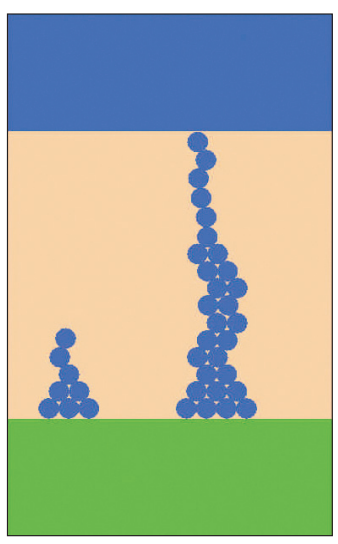

VCM

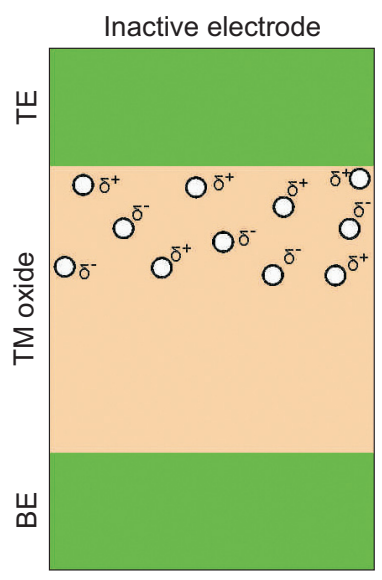

Inactive electrode

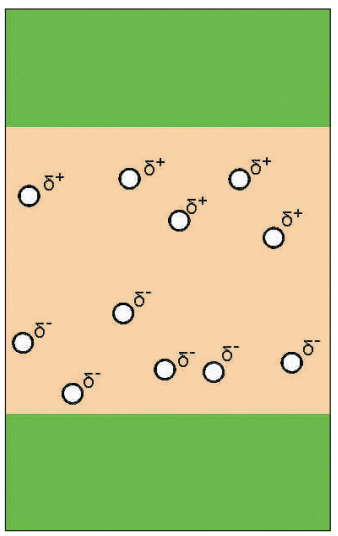

PCM

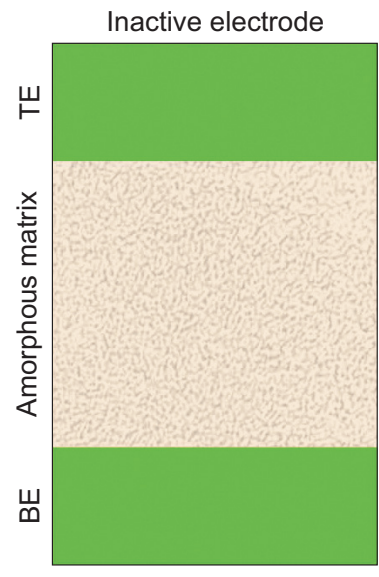

Inactive electrode

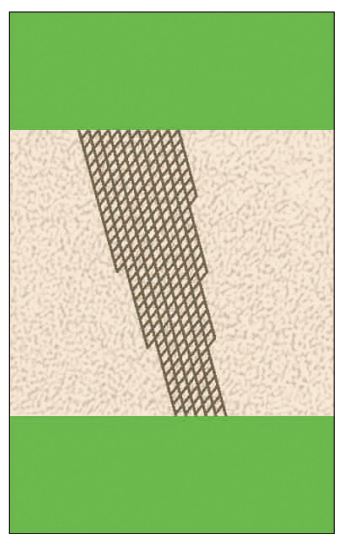

Fig. 1. Three different types of memristive devices whose analysis is being covered in this communication and the difference between their high (Off) and low (On) की resistance states. In electrochemical $\underset{\mathbb{D}}{*}$ metallization (ECM) cells a conductive filament forms from what is coined the active electrode towards the inactive electrode. The active electrode and its conductive filament consists of non-inert metals like $\mathrm{Ag}$ or $\mathrm{Cu}$. Inactive electrodes in all these devices can consist of noble metals such as Au and Pt or of conductive compounds e.g., TiN. In valence change memory (VCM) cells transition metal (TM) oxides between the electrodes locally change their oxidation state by movement of charged defects, such as oxygen vacancies. This behaviour can occur in the form of homogeneous drift of 글 charge carriers or filamentary switching. of phase change memory (PCM) devices $\stackrel{\mathbb{D}}{\mathbb{D}}$ exhibit a phase change in the amorphous matrix where a crystalline conductive filament is formed. This behaviour can be observed in chalcogenides and e.g. in an amorphous $\mathrm{TiO}_{\mathrm{x}}$ where crystalline Magnéli filaments form. BE, bottom electrode; TE, top electrode. 
change memory (VCM) cells, phase change memory (PCM) and other less abundant devices like thermochemical memory (TCM) cells (Waser, 2012). The first three (ECM, PCM, and VCM) cells are all in the focus of intense research and the experimental possibilities of transmission electron microscopy with respect to these cells shall be discussed.

\section{SPECIFIC CHALLENGES}

\section{TEM Sample Preparation}

It is well known that artifact-free sample preparation for TEM is a big technological challenge. It can generally be divided into (1) location specific preparation where a certain area of interest from the "bulk" sample material is chosen to be prepared and (2) location unspecific preparation where a mostly uniform sample undergoes preparation without the need for choosing a specific feature of the sample. While both of these present their own challenges, location specific preparation is often needed for memristor samples. This need is driven by the aim to fabricate increasingly smaller devices as they generally exhibit lower power consumption which is crucial to many memristor applications. Typically, relating electrical properties with micro- or nanoscopic features is only feasible with devices of defined dimensions. As these range in size from a few hundred to below one micrometer, location unspecific sample preparation, e.g., by mechanical grinding or microtome cutting, is frequently not meaningful. One exception presents the cross-sectional "sandwich" preparation with a precision ion polishing system (PIPS) which, at least for laterally large samples, can be useful. In all cases the sample preparation techniques have to be critically scrutinized with respect to possible preparation artifacts and alterations to the sample.

Aside the question of location specific or unspecific preparation, the specific challenges of in situ or ex situ analysis are crucial. The former requires much more advanced preparation strategies because the sample must not only be sufficiently thin but also electrical contacts have to be forged without contaminating, damaging or short-circuiting the sample. If only the static On or Off states are to be analyzed, ex situ investigations offer an easily accessible, comparably fast and-through decades of experience with comparable samples-well established methodology. However, as the demand for highest possible accuracy has increased so has the demand for the highest possible sample quality. Spatial and energy resolution in spectroscopic investigations increases as the sample thickness decreases. In practice, this implies that the sample must be much thinner than one mean free path of the incident electrons to avoid phenomena caused by multiple scattering events as much as possible. Especially for accurate EELS analysis this means that samples below $50 \mathrm{~nm}$ thickness are preferable, particularly for quantification of results. In the following, typical sample preparation challenges are being discussed, with respect to memristor-specific questions and the aforementioned challenges.

\section{Location specific sample preparation by focused ion beam techniques}

Sample preparation by focused ion beam (FIB) is one of the most prevalent preparation techniques in the TEM community as it offers unique advantages over other methods (Giannuzzi \& Stevie, 1999; Langford \& Clinton, 2004; Tseng, 2004). For memristors-and other microelectronic deviceslocation specific preparation is frequently inevitable because the regions of interest are much smaller than what can be handled with conventional techniques, such as microtome cutting, dimpling and Argon ion thinning, or wet-etching. This is especially the case for junctions optimized for lowest possible power consumption, as small contacts generally represent higher resistance and thus operation at lower currents. Accordingly, less power is consumed due to reduced thermal losses. Examples for such devices are memristive tunnel junctions (Hansen et al., 2015) and a plethora of other devices integrated on wafer-scale which aim for CMOS compatibility (Kim et al., 2012; Xia et al., 2009).

Their laterally sub-micron dimensions aside, particularly the example of memristive tunnel junctions also exhibits layers with a thickness well below $10 \mathrm{~nm}$. FIB sample preparation can potentially alter layers through a variety of ion beaminduced artifacts, such as Ga implantation, amorphization of surface layers and altering the composition through redeposition (Kato, 2004; Mayer et al., 2007). Therefore, thinning samples from the backside (Giannuzzi, 2012; Kang et al., 2010)-i.e., with the beam propagating firstly through the wafer (backside) and subsequently through the layers of interest and lastly through the former surface layers is

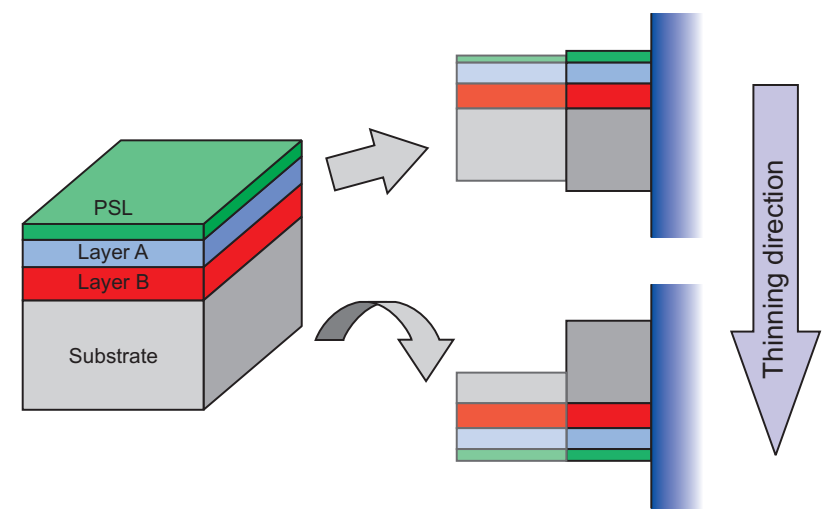

Fig. 2. Illustration of the preparation of a bi-layer sample via focused ion beam-scanning electron microscope: either it is being thinned from the protective surface layer (PSL) to the bottom or vice versa. The substratetypically a $\mathrm{Si}$ or $\mathrm{Si} / \mathrm{SiO}_{2}$ wafer-offers much higher protection and prevents artifacts like curtaining due to its inherent homogeneity. 
advisable (cf. Fig. 2). Another variation of this technique which is particularly interesting for nanostructures, is the "shadow-FIB" geometry (Vieweg et al., 2012) where also the substrate serves as the protective surface layer (PSL) during thinning of the regions of interest on top.

As the substrate is much thicker than any PSL (Pt in most cases) and has a vastly better homogeneity not only are the layers of interest better protected but thinning is much more uniform across the sample (Munroe, 2009). Another advantage is a shorter overall preparation time, because the deposition of a PSL can take several hours and its thickness can be drastically reduced from several micrometers to as thin as only about one micrometer. However, standard dualbeam FIB setups are rarely equipped with the mandatory equipment-a so called flip-stage which allows the sample to be turned upside down. In any case, one should consider the energy transferred to the specimen during sample preparation (Ishitani \& Yaguchi, 1996). Any small amount of energy brought into the sample in the form of heat or ballistic impact of the ion beam could potentially alter the memory cell overall or at least its resistance state. Cross-correlation experiments with different preparation techniques can help to identify clearly artifacts. Another possibility to exclude preparation artifacts is by a combined approach of TEM investigations with preparation free nanoanalytical techniques, such as $\mathrm{X}$-ray photoelectron spectroscopy (XPS) or advanced X-ray diffraction (XRD) analysis.

The described sample preparation methods is not, however, suitable for samples relying on filamentary switching as the specific areas where such filaments exist cannot be determined in the scanning electron microscope (SEM), so sample preparation would essentially become gambling.

\section{Location unspecific sample preparation}

In contrast to location specific preparation, location unspecific sample preparation is suitable for larger samples with specimen dimensions on the millimeter scale. Layerbased samples may be prepared by cross-sectional PIPS preparation; other techniques might be especially useful for devices that are not layer-based, like memristors made from nanostructures, such as single nanorods or -wires as these are representing easy to prepare horizontal devices (Kim et al., 2012; Liang et al., 2014; Yan et al., 2011). Investigations on such memristors are further discussed in section III-A-3. In the case of single nanowire samples the application of micro manipulators, e.g., in the SEM, is usually required. If the structures to be analyzed are large enough to be seen under light microscopes, careful manual handling and placement might be possible. To allow for in situ investigations or preand post-operation analysis without removing the specimen from the electron transparent TEM grid, samples have to be carefully placed between previously deposited electrodes which can be biased either in situ or ex situ depending on the available equipment. The need for carefully placing the samples is unnecessary if e.g. entire networks of switchable structures are used. They can frequently be deposited right on top of electron transparent membranes as parts of TEM sample grids without any need for careful placement (Avizienis et al., 2012; Liang et al., 2014; Sun et al., 2007). Alternatively, the nanowires can be randomly dispersed and the electrodes are deposited and shaped in a later stage with lithographic means (Chiang et al., 2011).

\section{Dedicated Techniques and Instrumentation}

Generally, standard investigations via HRTEM or SAED are not sufficient in order to characterize the small scale structures of memristive devices in depth. In order to resolve fine details in samples where the local chemistry is relevant, simultaneous sub-Ångstrom and sub-eV resolution of the electron beam have to be established, hence also setting high standards for experimental equipment. As beam damage can be easily induced, either beam currents, dwelling times or both have to be kept as low as possible, in turn requiring high collection yields from detectors and deft data processing to extract relevant signals over the ubiquitous noise. Especially for low signal-to-noise-ratio (SNR) data, e.g. fine structure signals in EELS, this represents a major challenge in the way of extracting the desired information. The demands for sample preparation are equally high. In order to extract information from the energy loss near-edge structure (ELNES) on chemical bonding or the extended energy loss fine structure (EXELFS) region on local chemical surrounding, samples have to be considerably thinner than $50 \mathrm{~nm}$ to minimize plural scattering and thus ease deconvolution as much as possible. The energy resolution should be at least $0.5 \mathrm{eV}$ in order to reveal fine details like the peak splitting in transition metal $\mathrm{L}$ and $\mathrm{M}$ edges. A number of publications can give further insight into the topic of electron spectroscopy (Egerton, 2007, 2011) and its application in combination with STEM (Pennycook et al., 2011).

Besides research on memristive materials, surveying research on other-in the broadest sense-electrical systems can help developing new techniques for memristive research as they face similar challenges especially in the case of in situ investigations. These systems include for example battery materials (Hammad Fawey et al., 2016) where highly advanced FIB techniques have been used for both thinning the regions of interest as well as forging the electrical contacts for in situ biasing, or supercapacitors (Ilari et al., 2016) where interfaces have been analyzed by EELS during and after in situ heating.

\section{SELECTED EXAMPLES}

\section{Filamentary Switching in Memristors}

Local filamentary switching is typically energetically favored 
over switching mechanisms governed by finely dispersed bulk diffusion because of lower interface energies and high affinity of like species to one another. Due to the required electroforming process (initial formation of filaments), filamentary switching is generally not the aspired outcome, instead bulk switching modes without an electroforming process are aimed at. In some cases filamentary switching is the desired outcome; in most reported cases, however, bulk switching without high-energy electroforming steps is favored. In this chapter the analysis of filamentary switching shall be highlighted by representative examples.

\section{Filaments through phase transformations}

Filaments standing out by a different crystal structure than their surrounding are most prominent in thick amorphous layers from which various crystalline strands can form. Best known for this behavior are Magnéli phases forming from an amorphous TiOx matrix (Kwon et al., 2010, cf. Fig. 3). Local peaks in electric field strength caused by imperfect electrodes induce the formation of oxygen deficient crystalline Tioxide filaments in these PCM cells. Characterization of these filaments is of course possible by quantification of the titanium to oxygen ratio, and also by structural determination. Other systems where such a behavior has been observed are $\mathrm{ZnO}$ (Chang et al., 2008; Chen et al., 2013; Huang et al., 2012, 2013; Song et al., 2011; Yao et al., 2012), NiO (Seo et al., 2004), $\mathrm{CuO}$ (Dong et al., 2007), $\mathrm{HfO}_{2}$ (Wu et al., 2011), and $\mathrm{SrTiO}_{3}$ (Szot et al., 2006). It should be noted here, that the distinction between a phase change in PCM cells is not only caused by diffusion-less phase changes but can also include diffusion, e.g. by oxygen and/or vacancies. This diffusion can result in phase changes between amorphous and crystalline phases in oxides (Kwon et al., 2010) or heavier chalcogenides (Kim et al., 2011; Li et al., 2013; Pino et al., 2010; Zalden et al., 2016), formation or alteration of local defects (Seo et al., 2004; Szot et al., 2006) or even a crystalline-crystalline transformation (Dong et al.,
2007).

If not by electrode imperfection, local peaks in electric field strength can manually be induced by application of scanning tunneling microscopy-TEM (STM-TEM) holders incorporating a movable STM needle-tip which can be biased with respect to the sample. This approach is certainly advantageous over trying to find filaments which have been induced ex situ because it is basically a needle-in-ahaystack problem as pre-grown filaments are normally not visible during sample preparation. This can be solved by either preparing samples with a high density of filaments or preparing many samples until a filament is found.

Kwon et al. (2010) characterized Magnéli filaments by application of such a highly sophisticated STM-TEM holder, successfully showing the formation and rupture of the crystalline filaments while simultaneously recording the I-V curves. However, as Strachan et al. (2010, 2013) demonstrated, the need for special equipment can be circumvented by clever sample design. Instead of the two previously mentioned approaches-needle-in-a-haystack and in situ-they fabricated a vertical crossbar array directly onto an electron transparent $\mathrm{Si}_{3} \mathrm{~N}_{4}$ membrane creating a stack with a combined thickness of around $100 \mathrm{~nm}$. While this represents the upper limit of specimen thickness that still allows effective analysis in the TEM it does obviate the need for both the special TEM holder as well as dedicated sample preparation. Furthermore, ex situ analysis can be undertaken because the sample must be biased outside the microscope but this can be done directly on the TEM grid without any intermediate sample preparation and thus without the possibility of artifact induction (Jang et al., 2016).

\section{Filaments through diffusion}

Contrary to PCM cells, resistive switching by diffusion of an active metal electrode into conducting filaments is observed in ECM cells. The problems arising for analyzing
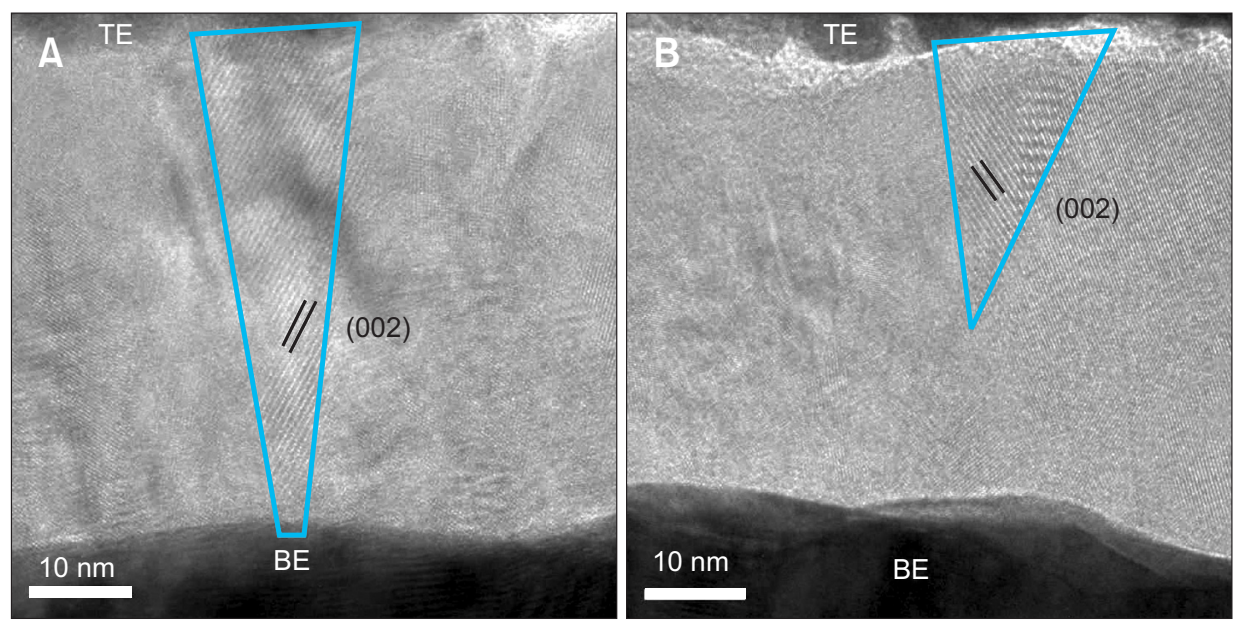

Fig. 3. Magnéli filaments in partially amorphous, partially polycrystalline mixed anatase and brookite structure $\mathrm{TiO}_{2}$ matrix as reported by Kwon et al. (2010). (A) $\mathrm{Ti}_{4} \mathrm{O}_{7}$ filament connecting top (TE) and bottom electrode (BE), (B) disconnected conical $\mathrm{Ti}_{4} \mathrm{O}_{7}$ filament. Reproduced with permission from ref. (Kwon et al., 2010). 
such devices resembles those discussed above for PCM cells closely. Standard approaches for sample preparation lead to a needle-in-a-haystack problem (Liu et al., 2010; Qian et al., 2016a), and inducing a filament in one certain location requires expensive and dedicated equipment. A crossbar like fabrication of samples for the previously discussed ex situ analysis is less advantageous though, because the electrode thickness has to be extremely small compared to real devices. The comparability between TEM samples and mass fabricated devices is likely not given; electrodes of only a few nanometer thickness-necessary to maintain electron transparencyrepresent an extremely small reservoir for forming the filaments. Fabricating thicker electrodes to overcome this problem would impede analysis by prohibiting transparency of the sample to the electron beam.

Another possibility for such a similar ex situ approach is pursued by Yang et al. (2012; cf. Fig. 4) Instead of a vertical crossbar array they demonstrated the possibility of producing horizontal nano gaps. These, too, have been fabricated directly onto a thin electron transparent silicon nitride membrane. The gap subsequently was filled with the electrolyte-chalcogenides in this case-necessary for silver ion movement. While this approach offers a fast and simple possibility to analyze the formation of silver filaments it might be inherently problematic: Surface diffusion occurs with vastly higher diffusion rates than bulk diffusion. As a horizontal stack is naturally thin (according to Yang et al. [2012] only $15 \mathrm{~nm}$ ) to allow for good electron transparency the surface dominated effects are dominant which are not relevant to the same extent for the devices. Rarely, this aspect is being discussed. Experiments to verify or rule out surface effects could include tomographic analysis where it is possible to determine whether the formed filaments or nanoparticle cluster (Guo et al., 2016) are within or above the matrix. Utilizing in situ approaches, live observation of filament growth is possible. This has been demonstrated in scientific publications numerous times (Chen et al., 2015; Choi et al., 2011; Liu et al., 2012; Tian et al., 2014). Alternatives to in situ TEM are for example presented by Di Martino et al. (2016).

\section{Memristive nanostructures}

Horizontal devices as discussed in the previous section can not only yield excellent TEM specific samples but are also pursued because of their inherent functionality. In terms of TEM analysis these devices are advantageous because they forestall the need for complicated and time-consuming crosssectional specimen preparation if deposited directly onto electron transparent membranes.

Horizontal memristive devices have been realized with switching nano-structures containing Ag wires (Avizienis et al., 2012), CuO nanowires (Fan et al., 2013; Liang et al., 2014), $\mathrm{ZnO}$ nanowires (Chiang et al., 2011), $\mathrm{TiO}_{2}$ (Lin et al., 2016), $\mathrm{Cu}_{2} \mathrm{~S}$ nanowires (Liu et al., 2015), GeTe nanowires (Sun et al., 2007), and Ge/Si nanowires (Yan et al., 2011). The large variety of material systems analyzed underlines the flexibility and relative ease with which such investigations can be conducted. Most of such experiments are performed while applying an electrical bias in situ so that the resistive switching can be observed dynamically. However, where appropriate equipment is lacking conducting pre- and post-operation analysis is a good and easy alternative.

\section{Homogenous Switching in Memristors}

\section{Anion movement based devices}

Devices making use of anion displacement rely on the change in conductivity linked with this movement and their change of resistance state is associated with a valence change. This can either be a macroscopic change in conducting paths or a laterally highly confined change in the case of tunnel junctions (Jeong et al., 2009, cf. Fig. 5). The observation of oxygen distribution in the memristive tunnel barrier allowed
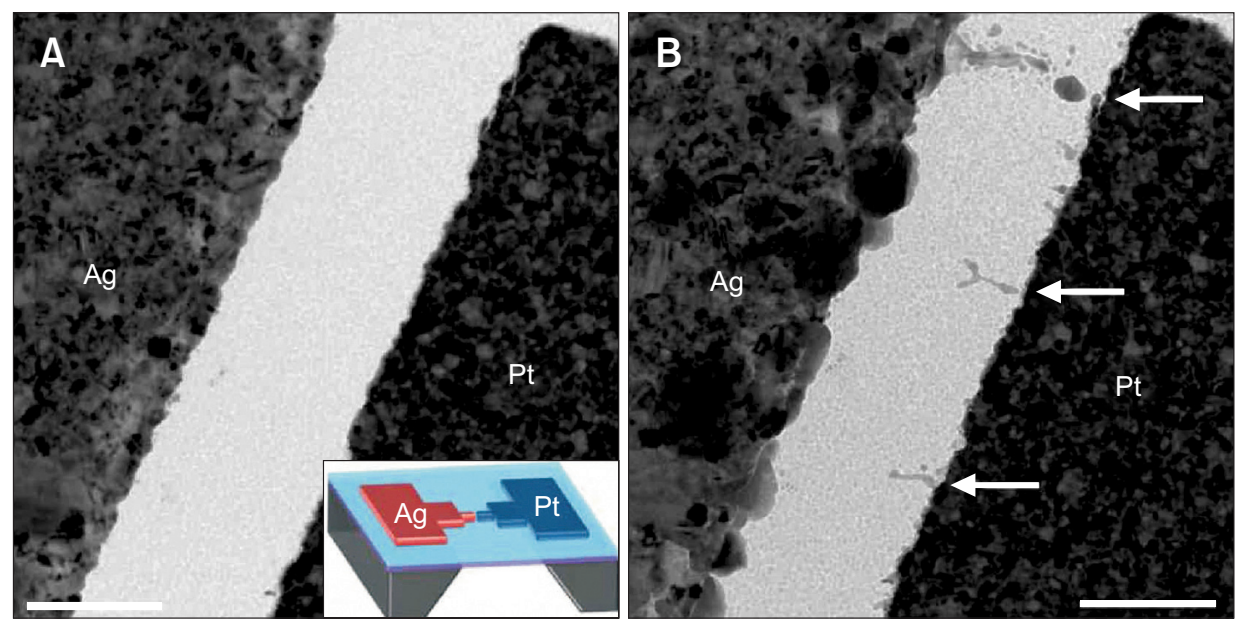

Fig. 4. Formation of conducting Ag filaments in an amorphous $\mathrm{SiO}_{2}$ matrix between an Ag and Pt electrode in horizontal configuration as reported by Yang et al. (2012). The device has been deposited directly onto an electron transparent $\mathrm{SiN}_{\mathrm{x}}$ membrane. Scale bar=200 nm. (A) Transmission electron microscope (TEM) image of the device as-fabricated before being biased. Inset is a sketch of the device design. (B) TEM image of the same device and same location after being biased outside the TEM. Conducting filaments formed during biasing are highlighted by arrows. Reproduced with permission from ref. (Yang et al., 2012). 
correlation of the Off state with a clearly segregated oxygen concentration profile whereas in the On state the oxygen is much more dispersed throughout the barrier.

Particularly in the latter case, determination of dielectric properties is required in order to describe and predict the electrical (tunneling) behavior of such devices. Especially determination of the full dielectric function-and thus the bandgap and relative permittivity-is one of the most pursued fields currently under development in the domain of electron microscopy which is only possible by application of valence EELS (VEELS). As the number of monochromatic TEMs rises, so does the availability of VEELS. However, even as disposability of equipment increases, interpretation of VEELS data is a major obstacle as relativistic effects-such as Cerenkov losses (Stöger-Pollach et al., 2006)-modify the VEEL spectrum especially in high relative permittivity (i.e., refractive index) materials (Stöger-Pollach \& Schattschneider, 2007). Also, the relevant loss signals lie in the tail of the zero loss peak (ZLP) and its correct subtraction is even more important than background removal is for core-loss EELS. This implies that the already non-straightforward application of EELS is even more intricate when it comes to accurate determination of dielectric properties. Furthermore, application of VEELS in STEM mode might be impossible altogether because of superposition of the inelastic signal with elastic momentumtransfer in the entire diffraction plane. This would limit the application of spatially resolved VEELS to TEM imaging mode, capturing several energy filtered micrographs and creating an image spectrum (as opposed to a STEM spectrum image).

However, instead of bandgap determination it is possible to find the oxidation state and the linked number of charge carriers by EELS. Specifically in highly ordered systems, such as epitaxial ferroelectric tunnel junctions (Chanthbouala et al., 2012) TEM investigations can yield information (a) by determining interface quality (Arita et al., 2016; Lee et al., 2016; Lin et al., 2008), (b) by testing for disordering, especially for charged defects which induce net polarization and thus have an effect on dielectric properties, and (c) by strain measurements (Vasudevan et al., 2014) which can cause domain pinning in ferroelectric layers.

In cases where it is not dielectric properties but rather spatially dependent conductivity, analysis of oxidation states and lateral distribution of anions, e.g. in bulk mixed ion conductors, can yield important information as they determine the number of available charge carriers or trap states (Privitera et al., 2013, 2015). Here, the resistance state of the memristor is defined by the oxygen or trap concentration profile; the ion conductivity is linearly dependent on mobility and concentration, the latter of which can be determined by EELS analysis of the metals oxidation state or even simpler but possibly less accurate by quantification of metal and oxygen contents from EDX data. For this, however, accurate quantification algorithms are necessary, e.g., $\zeta$-factor quantification.

As oxygen deficiency in otherwise stoichiometric compounds can also alter the structure substantially HRTEM investigations offer an easy way to visualize regions with different compositions-and thus different conductance-without the need for more complex spectroscopic investigations and equipment. This can be applied for example in $\mathrm{HfO}_{2}$ based switching layers (Calka et al., 2013) where stoichiometric crystalline regions are found right next to oxygen deficient amorphous regions. This behavior, however, is found rather rarely and for the sake of completeness a cross-correlation between structural and spectroscopic investigations is advisable. Nevertheless, as HRTEM and even more so electron diffraction experiments, normally require much lower exposure times and thus the energy input into the material is accordingly lower, such investigations offer an inherent advantage over spectroscopic analyses, where they are possible.

Furthermore, amorphous layers of only a few nanometer thickness, such as tunnel barriers can be characterized by their pair distribution function (PDF). For instance, the local PDF can be determined from nanobeam electron diffraction
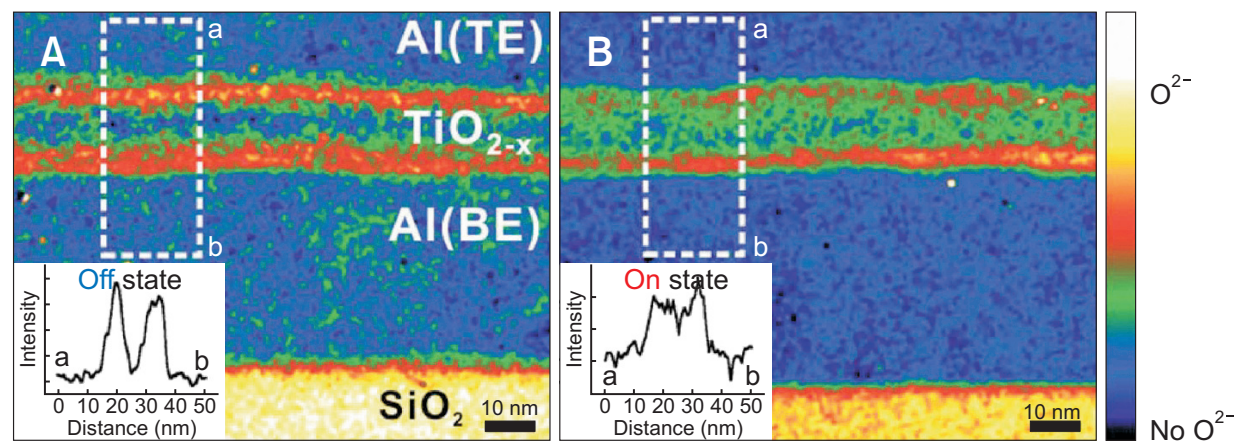

Fig. 5. Oxygen distribution in $\mathrm{TiO}_{2-\mathrm{x}}$ based tunnel barrier sandwiched between two $\mathrm{Al}$ electrodes as reported by Jeong et al. ranging from high oxygen content (bright yellow) to no detected oxygen (dark blue). (A) Oxygen ions segregate in the tunnel barrier at the two electrode interfaces thus forming the Off state. (B) Oxygen ions are finely dispersed throughout the tunnel barrier forming the On state. Insets show the integrated oxygen distribution in the area marked with white dashed lines. Reproduced with permission from ref. (Jeong et al., 2009). BE, bottom electrode; TE, top electrode. 
experiments (Hirotsu et al., 2001).

\section{Cation diffusion in thick layers}

Instead of anion movement it is also possible to manufacture devices with homogenous movement of metal ions without the formation of filaments. Such systems require information by means of tomography in order to visualize particle distribution and track diffusion. This in turn allows reconstruction of electrical fields (by simulation) and thus information about the driving forces of cation movement. Additionally, locating the different metal species can also yield information by comparing high and low resistance states.

For mixed ionic conductors the conductive properties have been exploited to serve as a memristive material such as in $\mathrm{GaOx}$ (Aoki et al., 2014). However, devices relying on cation movement tend to form conducting filaments instead of homogenous switching, as summarized by Valov and Kozicki (2013) in their review article. The example presented by Aoki et al. (2014) represents an exception and as apparent from their results, the change in $\mathrm{Ga}$ and $\mathrm{O}$ concentration profiles between On and Off state seems almost impossible to detect.

\section{OUTLOOK}

Besides the samples discussed in the preceding sections, other developments in the field of microelectronics are foreseeable. Lately, manufacturers of consumer electronics have begun developing prototypes relying on bendable soft matter electronics, especially for handheld devices such as smartphones. These, much like liquid crystal semiconductor systems or other organic samples, are usually not ideal for TEM analysis. However, as the quality of electromagnetic lenses improves, field emission guns offer higher-quality electron probes and aberration correctors become more and more abundant. Thus the accelerating voltage and electron dose can be reduced while maintaining a decent resolution. Likewise, the signal-to-noise ratio for spectroscopic methods has become better because of more efficient detectors thus allowing for lower beam currents and concordantly dose rates or energy input into the sample. Both developments allow the investigation of organic matter such as organic light emitting diodes (OLEDs) and the aforementioned bendable displays and organic-inorganic hybrids (Jeong et al., 2010; Kimura et al., 2012; Koo et al., 2011; Qian et al., 2016b). If these trends prove to be persistent, fabrication of organic matter memristors is very likely and analysis of these can advance in the same manner as for the examples above. Further miniaturization might even lead to memristors with a quantized conductance because dimensions of the conductor are reduced to a single row of atoms (Ohnishi et al., 1998). In situ TEM studies also offer possibilities towards understanding the underlying phenomena. However, this is completely dependent on the system employed, where challenges like insufficient resolution, sample preparation artefacts, electron beam induced sample modification may arise. Moreover differentiating beam induced change vs change resulting from electrical biasing is critical. On top of that, kinetic processes interrelated to oxygen mobility are difficult to measure during an in situ TEM experiment in real time due to insufficient time resolution.

Another certainly noteworthy aspect is the correlation of TEM results with atomic scale simulations such as kinetic Monte Carlo (Dirkmann et al., 2016; Toufik et al., 2015) or molecular dynamics (Savel'ev et al., 2011) simulations. They are powerful tools allowing for emulation of resistive switching phenomena on different timescales. Similar to structure or diffraction pattern simulations used when evaluating high resolution or diffraction experiments they give hints as to how the device changed from the ON to OFF states. As for most simulations and fitting algorithms these simulations suffer heavily under the start value problem; the more accurate the input-i.e., the initial pre-operation state-can be worked into the simulation the better and more accurate the result. Thus, simulations do not directly allow for evaluation of TEM investigations but instead help understanding switching mechanisms.

Lastly, software developments should not be underestimated. Image processing and multidimensional data analysis software like Hyperspy (Peña et al., 2016) advanced greatly, and is more easily available to the majority of scientists. They allow for analysis not only to speed up, but mathematical operations like deconvolution-removal of plural scattering effects from EEL spectra-and precise fitting with reference spectra also allow for a more accurate localization and identification of chemical species.

\section{CONCLUSIONS}

In summary, this comprehensive review of electron microscopy methods for nanoscale memristive or resistive switching devices highlights different possibilities to gather crucial information on switching mechanisms by observing $\mathrm{ON}$ and OFF states. Compared with in situ experiments, ex situ analysis in TEMs is easier to conceive and interpret and less prone to erroneous results. Typical pitfalls, such as the general discrepancy between TEM examinable specimen and "actual" implemented devices as well as hardly foreseeable effects such as surface migration have been discussed. Despite these possible obstructions, TEM analysis proves to be an extremely versatile analytical tool for both filamentary and bulk switching mechanism based devices.

Filamentary devices are best characterized by horizontal arrangement of electrodes and switching layers. The otherwise necessary cross sectional preparation has hardly any means of 
effectively localizing the position of filaments and selectively preparing a TEM suitable specimen.

Bulk switching based devices, because of their homogeneity of congeneric alteration in the memristive layer are comparably easy to handle, because cross sectional preparation does not require a specific location to be prepared for TEM investigation.

\section{CONFLICT OF INTEREST}

No potential conflict of interest relevant to this article was reported.

\section{ACKNOWLEDGMENTS}

The authors acknowledge funding through the Deutsche Forschunsgemeinschaft (DFG) through the research unit FOR2093 (project B2) "Memristive Bauelemente für neuronale Systeme" (Memristive devices for neuronal systems). The authors would like to thank Dr. Christian Kübel for valuable discussions regarding in situ aspects of analysis. The authors acknowledge the support of the Karlsruhe Nano Micro Facility for electron microscopy and spectroscopy.

\section{REFERENCES}

Aoki Y, Wiemann C, Feyer V, Kim H S, Schneider C M, III-Yoo H, and Martin M (2014) Bulk mixed ion electron conduction in amorphous gallium oxide causes memristive behaviour. Nat. Commun. 5, 3473.

Arita M, Ohno Y, and Takahashi Y (2016) Switching of Cu/MoOx/TiN CBRAM at MoOx/TiN interface. Phys. Status Solidi A 213, 306-310.

Avizienis A V, Sillin H O, Martin-Olmos C, Shieh H H, Aono M, Stieg A Z, and Gimzewski J K (2012) Neuromorphic atomic switch networks. PLoS One 7, e42772.

Calka P, Martinez E, Delaye V, Lafond D, Audoit G, Mariolle D, Chevalier N, Grampeix H, Cagli C, Jousseaume V, and Guedj C (2013) Chemical and structural properties of conducting nanofilaments in TiN/HfO2based resistive switching structures. Nanotechnology 24, 85706.

Chang W Y, Lai Y C, Wu T B, Wang S F, Chen F, and Tsai M J (2008) Unipolar resistive switching characteristics of $\mathrm{ZnO}$ thin films for nonvolatile memory applications. Appl. Phys. Lett. 92, 22110.

Chanthbouala A, Garcia V, Cherifi R O, Bouzehouane K, Fusil S, Moya X, Xavier S, Yamada H, Deranlot C, Mathur N D, Bibes M, Barthélémy A, and Grollier J (2012) A ferroelectric memristor. Nat. Mater. 11, 860864.

Chen J Y, Hsin C L, Huang C W, Chiu C H, Huang Y T, Lin S J, Wu W W, and Chen L J (2013) Dynamic evolution of conducting nanofilament in resistive switching memories. Nano Lett. 13, 3671-3677.

Chen J Y, Huang C W, Chiu C H, Huang Y T, and Wu W W (2015) Switching kinetic of VCM-based memristor: evolution and positioning of nanofilament. Adv. Mater. 27, 5028-5033.

Chiang Y D, Chang W Y, Ho C Y, Chen C Y, Ho C H, Lin S J, Wu T B, and He J H (2011) Single-ZnO-nanowire memory. IEEE Trans. Electron Devices 58, 1735-1740.

Choi S J, Park G S, Kim K H, Cho S, Yang W Y, Li X S, Moon J H, Lee K J, and Kim K (2011) In situ observation of voltage-induced multilevel resistive switching in solid electrolyte memory. Adv. Mater. 23, 32723277.

Di Martino G, Tappertzhofen S, Hofmann S, and Baumberg J (2016) Nanoscale plasmon-enhanced spectroscopy in memristive switches. Small 12, 1334-1341.

Dirkmann S, Hansen M, Ziegler M, Kohlstedt H, and Mussenbrock T (2016) The role of ion transport phenomena in memristive double barrier devices. Sci. Rep. 6, 35686.

Egerton R F (2007) Limits to the spatial, energy and momentum resolution of electron energy-loss spectroscopy. Ultramicroscopy
107, 575-586.

Egerton R F (2011) Electron Energy-Loss Spectroscopy in the Electron Microscope (Springer Science \& Business Media, New York).

Fan Z, Fan X, Li A, and Dong L (2013) In situ forming, characterization, and transduction of nanowire memristors. Nanoscale 5, 1231012315.

Giannuzzi L A (2012) Routine backside FIB milling with EXpressLO. In: Proceedings from ISTFA 2012, pp. 388-390, (ASM International, Materials Park).

Giannuzzi L A and Stevie F A (1999) A review of focused ion beam milling techniques for TEM specimen preparation. Micron 30, 197-204.

Guo A, Li D, Li W, Gu D, Jiang X, and Jiang Y (2016) The relation of structure and dispersion to amorphous silicon silver thin films. Mater. Lett. 185, 5-8.

Hammad Fawey M, Chakravadhanula V S K, Reddy M A, Rongeat C, Scherer T, Hahn H, Fichtner M, and Kübel C (2016) In situ TEM studies of micron-sized all-solid-state fluoride ion batteries: preparation, prospects, and challenges. Microsc. Res. Tech. 79, 615624.

Hansen M, Ziegler M, Kolberg L, Soni R, Dirkmann S, Mussenbrock T, and Kohlstedt H (2015) A double barrier memristive device. Sci. Rep. 5, 13753.

Hasegawa T, Terabe K, Tsuruoka T, and Aono M (2012) Atomic switch: atom/ion movement controlled devices for beyond von-neumann computers. Adv. Mater. 24, 252-267.

Hirotsu Y, Ishimaru M, Ohkubo T, Hanada T, and Sugiyama M (2001) Application of nano-diffraction to local atomic distribution function analysis of amorphous materials. J. Electron Microsc. 50, 435-442.

Huang C H, Huang J S, Lin S M, Chang W Y, He J H, and Chueh Y L (2012) $\mathrm{ZnO}_{1-\mathrm{x}}$ nanorod arrays/ZnO thin film bilayer structure: from homojunction diode and high-performance memristor to complementary 1D1R application. ACS Nano 6, 8407-8414.

Huang Y T, Yu S Y, Hsin C L, Huang C W, Kang C F, Chu F H, Chen J Y, Hu J C, Chen L T, He J H, and Wu W W (2013) In situ TEM and energy dispersion spectrometer analysis of chemical composition change in $\mathrm{ZnO}$ nanowire resistive memories. Anal. Chem. 85, 3955-3960.

Ilari G M, Chawla V, Matam S, Zhang Y, Michler J, and Erni R (2016) Electron energy loss spectroscopy analysis of the interaction of $\mathrm{Cr}$ and V with MWCNTs. Micron 84, 37-42.

Ishitani T and Yaguchi T (1996) Cross-sectional sample preparation by 
focused ion beam: a review of ion-sample interaction. Microsc. Res. Tech. 35, 320-333.

Jang M H, Agarwal R, Nukala P, Choi D, Johnson A T C, Chen I W, and Agarwal R (2016) Observing oxygen vacancy driven electroforming in Pt-TiO2-Pt device via strong metal support interaction. Nano Lett. 16, 2139-2144.

Jeong H Y, Kim J Y, Kim J W, Hwang J O, Kim J E, Lee J Y, Yoon T H, Cho B J, Kim S O, Ruoff R S, and Choi S Y (2010) Graphene oxide thin films for flexible nonvolatile memory applications. Nano Lett. 10, 43814386.

Jeong H Y, Lee J Y, Choi S Y, and Kim J W (2009) Microscopic origin of bipolar resistive switching of nanoscale titanium oxide thin films. Appl. Phys. Lett. 95, 162108.

Kang H J, Kim J H, Oh J W, Back T S, and Kim H J (2010) Ultra-thin TEM sample preparation with advanced backside FIB milling method. Microsc. Microanal. 16, 170-171.

Kato N I (2004) Reducing focused ion beam damage to transmission electron microscopy samples. J. Electron Microsc. 53, 451-458.

Kim K H, Gaba S, Wheeler D, Cruz-Albrecht J M, Hussain T, Srinivasa N, and Lu W (2012) A functional hybrid memristor crossbar-array/CMOS System for data storage and neuromorphic applications. Nano Lett. 12, 389-395.

Kim S, Park J, Jung S, Lee W, Woo J, Cho C, Siddik M, Shin J, Park S, Lee $\mathrm{B} \mathrm{H}$, and Hwang $\mathrm{H}$ (2011) Excellent resistive switching in nitrogendoped Ge2Sb2Te5 devices for field-programmable gate array configurations. Appl. Phys. Lett. 99, 192110.

Kimura M, Honda K, Yodogawa S, Ohtsuka K, Oo T N, Miyashita K, Hirata $\mathrm{H}$, and Akahane T (2012) Flexible LCDs fabricated with a slit coater: not requiring an alignment film. J. Soc. Inf. Disp. 20, 633-639.

Koo H J, So J H, Dickey M D, and Velev O D (2011) Towards all-soft matter circuits: prototypes of quasi-liquid devices with memristor characteristics. Adv. Mater. 23, 3559-3564.

Kwon D H, Kim K M, Jang J H, Jeon J M, Lee M H, Kim G H, Li X S, Park G S, Lee B, Han S, Kim M, and Hwang C S (2010) Atomic structure of conducting nanofilaments in $\mathrm{TiO} 2$ resistive switching memory. Nat. Nanotechnol. 5, 148-153.

Langford R M and Clinton C (2004) In situ lift-out using a FIB-SEM system. Micron 35, 607-611.

Lee A R, Baek G H, Kim T Y, Ko W B, Yang S M, Kim J, Im H S, and Hong J P (2016) Memory window engineering of Ta205-x oxide-based resistive switches via incorporation of various insulating frames. Sci. Rep. 6, 30333.

Li Y, Zhong Y, Xu L, Zhang J, Xu X, Sun H, and Miao X (2013) Ultrafast synaptic events in a chalcogenide memristor. Sci. Rep. 3, 1619.

Liang K D, Huang C H, Lai C C, Huang J S, Tsai H W, Wang Y C, Shih Y C, Chang M T, Lo S C, and Chueh Y L (2014) Single CuOx nanowire memristor: forming-free resistive switching behavior. ACS Appl. Mater. Interfaces 6, 16537-16544.

Lin C Y, Lee D Y, Wang S Y, Lin C C, and Tseng T Y (2008) Effect of thermal treatment on resistive switching characteristics in $\mathrm{Pt} / \mathrm{Ti} / \mathrm{Al}_{2} \mathrm{O}_{3} / \mathrm{Pt}$ devices. Surf. Coat. Technol. 203, 628-631.

Lin L, Liu L, Musselman K, Zou G, Duley W W, and Zhou Y N (2016) Plasmonic-radiation-enhanced metal oxide nanowire heterojunctions for controllable multilevel memory. Adv. Funct. Mater. 5979-5986.

Liu P H, Lin C C, Manekkathodi A, and Chen L J (2015) Multilevel resistance switching of individual Cu2S nanowires with inert electrodes. Nano Energy 15, 362-368.
Liu Q, Long S, Lv H, Wang W, Niu J, Huo Z, Chen J, and Liu M (2010) Controllable growth of nanoscale conductive filaments in solidelectrolyte-based ReRAM by using a metal nanocrystal covered bottom electrode. ACS Nano 4, 6162-6168.

Liu Q, Sun J, Lv H, Long S, Yin K, Wan N, Li Y, Sun L, and Liu M (2012) Real-time observation on dynamic growth/dissolution of conductive filaments in oxide-electrolyte-based ReRAM. Adv. Mater. 24, 18441849.

Mayer J, Giannuzzi L A, Kamino T, and Michael J (2007) TEM sample preparation and FIB-induced damage. MRS Bulletin 32, 400-407.

Munroe P R (2009) The application of focused ion beam microscopy in the material sciences. Mater. Charact. 60, 2-13.

Ohnishi H, Kondo Y, and Takayanagi K (1998) Quantized conductance through individual rows of suspended gold atoms. Nature $\mathbf{3 9 5}, 780-$ 783.

Peña F, Ostasevicius T, Fauske V T, Burdet P, Jokubauskas P, Sarahan M, Johnstone D, Nord M, Taillon J, Caron J, MacArthur K E, Eljarrat A, Mazzucco S, Furnival T, Prestat E, Walls M, Donval G, Martineau B, Zagonel L F, Garmannslund A, Aarholt T, Gohlke C, and iygr (2016) hyperspy: HyperSpy 1.1.

Pino R E, Bohl J W, McDonald N, Wysocki B, Rozwood P, Campbell K A, Oblea A, and Timilsina A (2010) Compact method for modeling and simulation of memristor devices: ion conductor chalcogenide-based memristor devices. In: 2010 IEEE/ACM International Symposium on Nanoscale Architectures, pp. 1-4, (IEEE).

Privitera S, Bersuker G, Butcher B, Kalantarian A, Lombardo S, Bongiorno C, Geer R, Gilmer D C, and Kirsch P D (2013) Microscopy study of the conductive filament in $\mathrm{HfO}_{2}$ resistive switching memory devices. Microelectron. Eng. 109, 75-78.

Privitera S, Bersuker G, Lombardo S, Bongiorno C, and Gilmer D C (2015) Conductive filament structure in $\mathrm{HfO}_{2}$ resistive switching memory devices. Solid-State Electron. 111, 161-165.

Qian K, Nguyen V C, Chen T, and Lee P S (2016a) Amorphous-Si-based resistive switching memories with highly reduced electroforming voltage and enlarged memory window. Adv. Electron. Mater. 2, 1500370.

Qian K, Tay R Y, Nguyen V C, Wang J, Cai G, Chen T, Teo E H T, and Lee P S (2016b) Hexagonal boron nitride thin film for flexible resistive memory applications. Adv. Funct. Mater. 26, 2176-2184.

Savel'ev S E, Alexandrov A S, Bratkovsky A M, and Williams R S (2011) Molecular dynamics simulations of oxide memristors: thermal effects. Appl. Phys. A: Mater. Sci. Process. 102, 891-895.

Seo S, Lee M J, Seo D H, Jeoung E J, Suh D S, Joung Y S, Yoo I K, Hwang I R, Kim S H, Byun I S, Kim J S, Choi J S, and Park B H (2004) Reproducible resistance switching in polycrystalline NiO films. Appl. Phys. Lett. 85, 5655-5657.

Song J, Zhang Y, Xu C, Wu W, and Wang Z L (2011) Polar charges induced electric hysteresis of $\mathrm{ZnO}$ nano/microwire for fast data storage. Nano Lett. 11, 2829-2834.

Stöger-Pollach M, Franco H, Schattschneider P, Lazar S, Schaffer B, Grogger W, and Zandbergen H W (2006) Cerenkov losses: a limit for bandgap determination and Kramers-Kronig analysis. Micron 37, 396-402.

Stöger-Pollach M, and Schattschneider P (2007) The influence of relativistic energy losses on bandgap determination using valence EELS. Ultramicroscopy 107, 1178-1185.

Strachan J P, Pickett M D, Yang J J, Aloni S, David Kilcoyne A L, MedeirosRibeiro G, and Stanley Williams R (2010) Direct identification of the 
conducting channels in a functioning memristive device. Adv. Mater. 22, 3573-3577.

Strachan J P, Yang J J, Montoro L A, Ospina C A, Ramirez A J, Kilcoyne A L D, Medeiros-Ribeiro G, and Williams R S (2013) Characterization of electroforming-free titanium dioxide memristors. Beilstein J. Nanotechnol. 4, 467-473.

Sun X, Yu B, Ng G, and Meyyappan M (2007) One-dimensional phasechange nanostructure: germanium telluride nanowire. J. Phys. Chem. C 111, 2421-2425.

Tian X, Wang L, Wei J, Yang S, Wang W, Xu Z, and Bai X (2014) Filament growth dynamics in solid electrolyte-based resistive memories revealed by in situ TEM. Nano Res. 7, 1065-1072.

Toufik S, Liping W, Louis G, and Asen A (2015) Physical simulation of sibased resistive randomaccess memory devices. In: Proceedings from SISPAD 2015, pp. 385-388, (IEEE).

Tseng A A (2004) Recent developments in micromilling using focused ion beam technology. J. Micromech. Microeng. 14, R15.

Valov I and Kozicki M N (2013) Cation-based resistance change memory. J. Phys. D: Appl. Phys. 46, 74005.

Vasudevan R K, Matsumoto Y, Cheng X, Imai A, Maruyama S, Xin H L, Okatan M B, Jesse S, Kalinin S V, and Nagarajan V (2014) Deterministic arbitrary switching of polarization in a ferroelectric thin film. Nat. Commun. 5, 4971.

Vieweg B F, Butz B, Peukert W, Klupp Taylor R N, and Spiecker E (2012) TEM preparation method for site- and orientation-specific sectioning of individual anisotropic nanoparticles based on shadow-FIB geometry. Ultramicroscopy 113, 165-170.
Waser R (2012) Redox-based resistive switching memories. J. Nanosci. Nanotechnol. 12, 7628-7640.

Wu X, Li K, Raghavan N, Bosman M, Wang Q X, Cha D, Zhang X X, and Pey $\mathrm{K} \mathrm{L}$ (2011) Uncorrelated multiple conductive filament nucleation and rupture in ultra-thin high-K dielectric based resistive random access memory. Appl. Phys. Lett. 99, 93502.

Xia Q (2011) Nanoscale resistive switches: devices, fabrication and integration. Appl. Phys. A: Mater. Sci. Process. 102, 955-965.

Xia Q, Robinett W, Cumbie M W, Banerjee N, Cardinali T J, Yang J J, Wu W, Li X, Tong W M, Strukov D B, Snider G S, Medeiros-Ribeiro G, and Williams R S (2009) Memristor-CMOS hybrid integrated circuits for reconfigurable logic. Nano Lett. 9, 3640-3645.

Yan H, Choe H S, Nam S, Hu Y, Das S, Klemic J F, Ellenbogen J C, and Lieber C M (2011) Programmable nanowire circuits for nanoprocessors. Nature 470, 240-244.

Yang Y, Gao P, Gaba S, Chang T, Pan X, and Lu W (2012) Observation of conducting filament growth in nanoscale resistive memories. Nat. Commun. 3, 732.

Yao I C, Lee D Y, Tseng T Y, and Lin P (2012) Fabrication and resistive switching characteristics of high compact Ga-doped ZnO nanorod thin film devices. Nanotechnology 23, 145201.

Zalden P, Shu M J, Chen F, Wu X, Zhu Y, Wen H, Johnston S, Shen Z X, Landreman P, Brongersma M, Fong S W, Wong H S P, Sher M J, Jost $P$, Kaes M, Salinga M, von Hoegen A, Wuttig M, and Lindenberg A M (2016) Picosecond electric-field-induced threshold switching in phase-change materials. Phys. Rev. Lett. 117, 67601. 